\title{
A Faraday Cup with High Frequency Response for a 200 MeV LINAC Proton Beam*
}

\author{
M. S. Zucker and J. W. Bittner \\ Brookhaven National Laboratory \\ Upton, NY 11973
}

\section{Abstract}

The purpose of this device, composed essentially of coaxial line elements, is monitoring, on a per micropulse basis, the beam intensity of a $200 \mathrm{MeV}$ LINAC at the BNL Radiation Effects Facility. The center conductor of the coaxial line acts as a beam stop. The output pulses are suitable for fast timing.

\section{MECHANICAL DESIGN AND CONSTRUCTION}

Figure 1 shows the general mechanical configuration of the Faraday Cup.

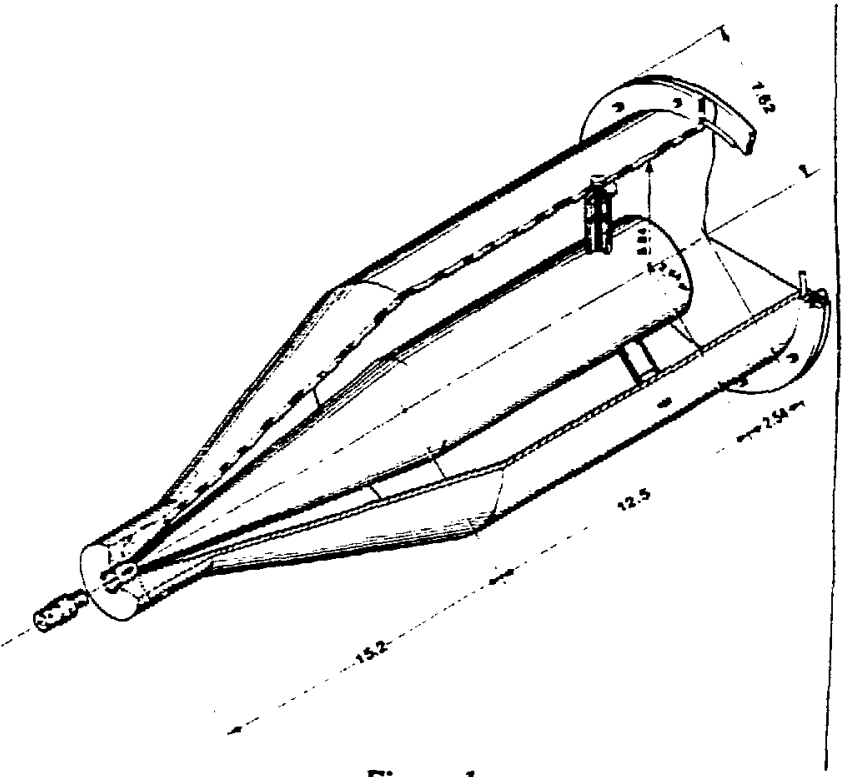

Figure 1.

Mechanical Configuration of the Faraday Cup

The front end of the center conductor, which acts as a beam stop, has to be range thick for $200 \mathrm{MeV}$ protons, bit as used in fast timing applications, requires only a modest lateral extent, since these beams are usually well-focused. A $5 \mathrm{~cm}$ diameter accommodates essentially all of a typical focused REF Gaussian beam profile (about $1 \mathrm{~cm}$ FWHM). The front of the beam stop is a right circular cylinder approximately range-thick in length, inside a cylindrical housing. The beam stop then tapers down, with a conical section forming the transition between the cylindrical portion of a $50 \mathrm{ohm}$ cable connector which is fastened to the back end of the housing. This tapered section (half-angle 9.46 degrees) and the similarly tapered housing in that region (half-angle 21.57 degrees) form a section of a conical transmission line with 50 ohm impedance.

The beam stop is pure aluminum to minimize artivation. The outer housing, normally struck only by stray or scattered beam, is hard aluminum for ease in machining and strength. The beam stop is mainly positioned relative to the outer housing by radial steatite insulators $120^{\circ}$ apart. There is also some support by the center conductor of the coaxial connector which threads into the narrow end of the beam stop. The front is closed with Al foil $(-.015 \mathrm{~cm})$ held in place between a flange on the outer housing and a clamping ring, using an O-ring to make the seal. The intent is to use this Faraday Cup either evacuated to a forepump pressure or to have an atmosphere of argon or air; a side tube welded to the body of the outer housing allows for evacuation and filling.

\section{ELECTRICAL DESIGN}

A bias voltage can be applied to the beam stop through the side leg of a $T$ introduced into the coaxial cable connected to the cup; the leg would have an isolation resistance in it to decouple the line from the bias source.

The high frequency design is based on two formulae for the characteristic impedance of, respectively, (circular cylindrical) coaxial, and bi-conical, transmission lines. The coaxial line impedance is given by $\mathrm{Z}_{0}=(1 / 2 \pi)(\mu / \epsilon)^{1 / 2} \ln (\mathrm{D} / \mathrm{d})$ where $\epsilon$ and $\mu$ are the dielectric constant and permeability of the medium between the conductors, and $D / d$ is the ratio of the outer to inner diameters of the concentric conductors. For $Z_{\mathrm{o}}=50 \mathrm{ohms}$, $D / d=2.303 \ldots$ The bi-conical line impedance is $Z_{0}=(1 / 2 \pi)(\mu / \epsilon)^{1 / 2} \ln \left\{\cot \left(\theta_{1} / 2\right) / \cot \left(\theta_{2} / 2\right)\right\}$ where $\theta_{1}$ and $\theta_{2}$ are half-angles of the inner and outer cones, see Figure $2^{1,2}$

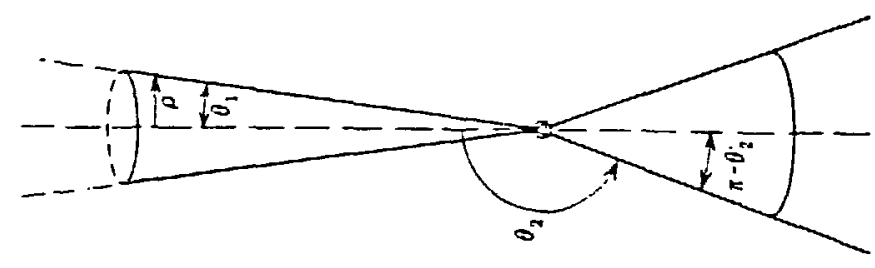

Figutre 2

The Geometry of a Conical Transmission Line 
This latter formula applied to matching the diameter of tive cylindrical portion of the beam stop to the coaxial cable connector led to the angular dimensions cited above. Each of these formulae strictly applies only to an infinite transmission line, however experieace shows that if transitions are not "too abrupt", reflections from these places will not be significant. In the interest of simplifying the machine shop work, no special attempt was made to make the transition any more gradual than dictated by the purely mechanical constraints of joining the basic $50 \mathrm{ohm}$ sections to one another. Ita fact, the only trouble in regard to impedance matching was experienced with a commercia? hermetic seal BNC style connector that deviated grossly from 50 obms. Replacing this with a modified type $N$ unit gave the good results to be shown below.

\section{TIME DOMAIN REFLECTOMETRY TESTS}

The Faraday Cup was tested by putting a step function pulse into the BNC connector and looking for reflections. Figure 3 shows such a trace.

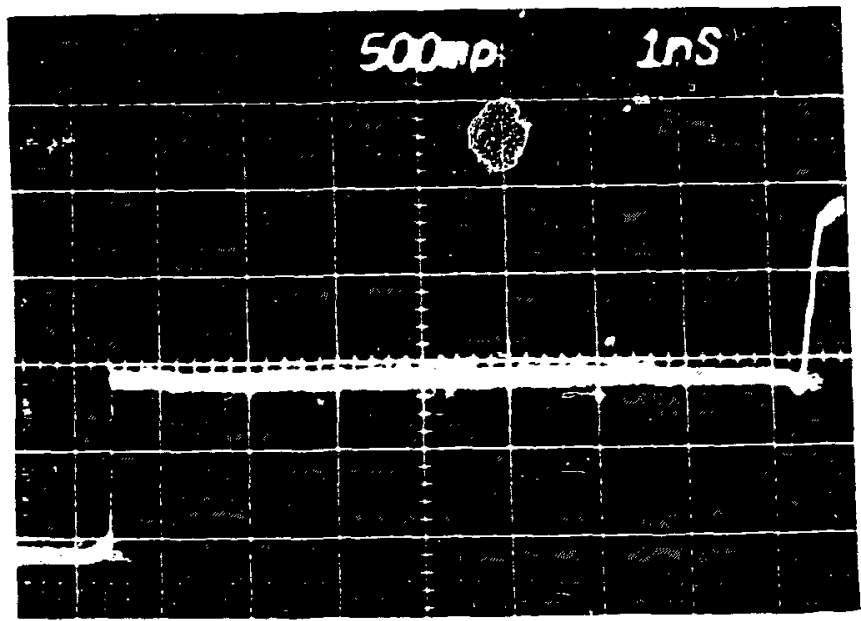

Figure 3

\section{A Time Domain Reflectometry Test of the Faraday Cup}

The vertical line on the left is the leading edge of the pulse put into a cable about 6 ns long connected to the Faraday Cup. The vertical line on the right represents the reflection from the end of the beam stop. The wiggles in the oscilloscope trace centered about $6 \mathrm{~cm}$ (6 $\mathrm{ns}$ ) to the right of the leading edge is caused by poor matching in the vicinity of the coax connector. These deviations from the flatness that would signify perfect matching were worse by a factor of about 5 before replacing the connector with the $N$ connector. The region between the wiggles at $6 \mathrm{~cm}$ and the vertical rise signifying the front end of the beam stop represents the matching behavior of the rest of the Faraday Cup.

\section{LINAC BEAM TESTS}

The Faraday Cup output signal witl: REF facility LINAC beam entering the front end was examined with a 1 GHZ real time oscilloscope. Figure 4 shows the envelope of a macropulse about $\mathbf{4 8 0}$ microseconds long.

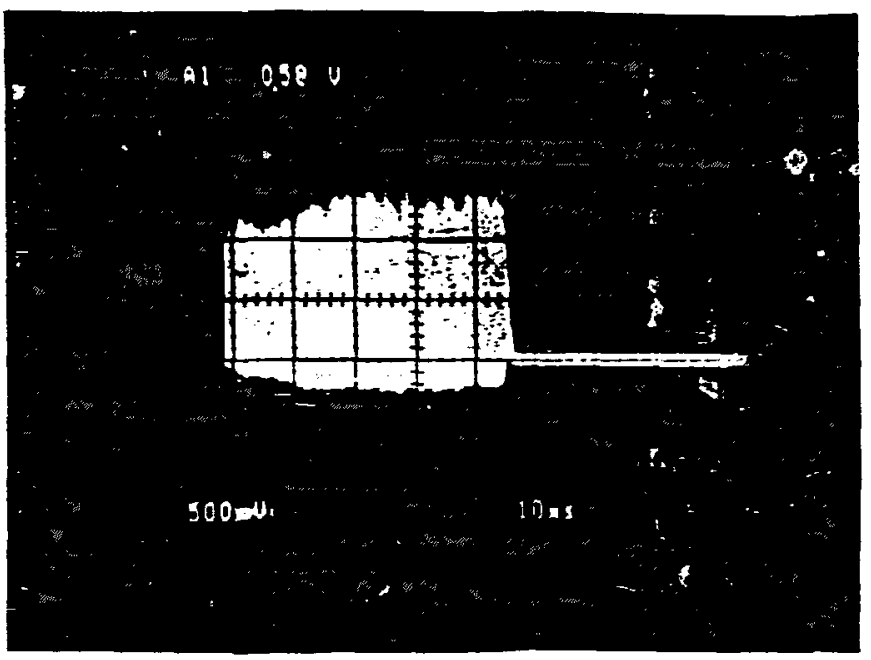

Figure 4

The Envelope of a (480 Microsecond) Macropulse

The envelope is greater than $1 \mathrm{~V}$ across 50 ohms, equivalent to $20 \mathrm{~mA}$ of beam current. This picture demonstrates the change of micropulse amplitude during the macropulse. Exparding the horizontal (time) axis to $2 \mathrm{~ns}$ per $\mathrm{cm}$ produced the trace in Figure 5, which shows some sections of the pulse train of individual micropulses superimposed on one another, since the oscilloscope beam made several sweeps during the film exposure. (This oscilloscope is capable of accurately displaying a Gaussian pulse shape with a FWHM equal to 0.5 ns.)

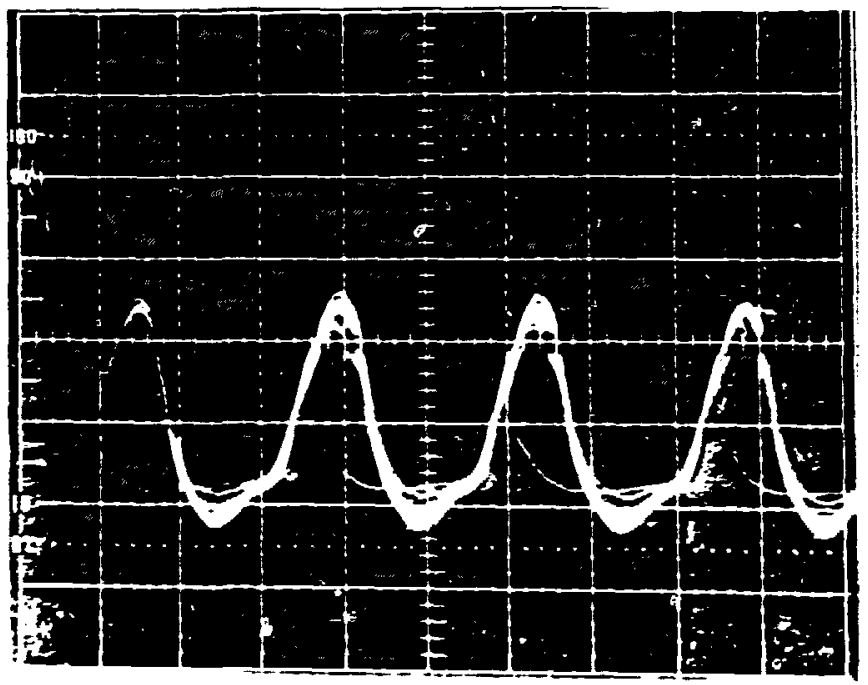

Figure 5

Individual Micropulses, Swecp Speed of $2 \mathrm{~ns}$ per $\mathrm{cm}$ 
The results are disappointing in that a micropulse as measured by this device is somewhat wider ( $1.5 \mathrm{~ns}$ FWHM) thar that measured by a wall gap monitor developed at the REF (about 1 ns FWHM). Another artifact that is not understood very well is the way the trace goes slightly negative. The only somewhat vague argument that can be mustered to explain this is that the assembly as a whole is acting as a cavity which is partially reacting to the shock excitation of the beam pulses by ringing a little.

\section{EVALUATION AND FUTURE DEVELOPMENT}

For the intended application, this Faraday Cup can be compared with a wall gap monitor. This device offers much the same facility as a wall gap monitor in that it sees the whole of the beam, and also has the frequency response to develop a pulse for each micropulse, and these pulses are as uniform in amplitude, presumably, as the envelope of the macropulse. The Faraday Cup is probably capable of a more accurate absolute beam current calibration than is the WGM, but must be placed after the target, which may lead in some cases to inaccuracy due to scattering of beam before the FC is reached. The wall gap monitor has to be installed in the beam pipe however, which require some effort every time the beam line is reconfigured to suite a particular experiment, while this device, being external to the vacuum system, is relatively easy to set up.

A possible course for future development would be to improve the matching at the cable-Faraday Cup interface, making larger diameter beam stops to accommodate less focused beams, and improving the vacuum capability.

\section{ACKNOWLEDGEMENTS}

The support and interest of $R$. Lankshear were important in accomplishing this project. The machine shop work was by W. Eberl.

\section{REFERENCES}

[1] W. R. Smythe, Static and Dynamic Electricity, 2nd edition, New York: McGraw-Hill Book Company, 1950, pp. 479-480.

[2] W. L. Weeks, Electromagnetic Theory for Engineering Applications, New York: John Wiley and Sons, 1964, pp. 530-533.

- This work was performed under the auspices of the U. S. Department of Energy under Contract No. DE-ACO2$76 \mathrm{CI} 100016$. 